\title{
Principal component analysis of MALDI TOF MS mass spectra separates $M$. abscessus (sensu stricto) from M. massiliense isolates
}

\author{
Jan Kehrmann ${ }^{1 *}$, Sarah Wessel', Roshni Murali ${ }^{1}$, Annegret Hampel ${ }^{2}$, Franz-Christoph Bange ${ }^{2}$, Jan Buer ${ }^{1}$ \\ and Frank Mosel $^{1}$
}

\begin{abstract}
Background: The discrimination of the members of the Mycobacterium abscessus complex is of clinical interest because one of the subspecies, M. massiliense, exhibits higher rates of response to antibiotic treatment for lung infection than do the other members of that complex. M. abscessus complex contains three subspecies that are laborious to identify; therefore, a routine diagnostic tool would be worthwhile.

Results: We used principal component analysis, hierarchical cluster analysis, and single-peak analysis to examine peak lists derived from matrix-assisted laser desorption/ionization time-of-flight mass spectrometry (MALDI TOF MS) mass spectra of 50 clinical M. abscessus complex isolates, including $28 \mathrm{M}$. abscessus (sensu stricto), 19 M. massiliense, and $3 \mathrm{M}$. bolletii isolates grown in mycobacterium growth indicator tube liquid medium and prepared with a bead-based protocol. Principal component analysis but not hierarchical cluster analysis separated M. abscessus (sensu stricto) isolates and M. massiliense isolates into two clusters. Furthermore, single-peak analysis displayed 4 discriminating peaks that separated M. abscessus (sensu stricto) from M. massiliense isolates. M. bolletii isolates did not exhibit specific peaks but resembled the M. abscessus (sensu stricto) peak profile and also grouped within this principal component analysis cluster. Principal component analysis of all peak lists with the exclusion of the four discriminating peaks again separated M. abscessus (sensu stricto) from M. massiliense isolates, thus relativizing the importance of these peaks for subspecies identification.
\end{abstract}

Conclusions: Principal component analysis of peak lists derived from MALDI TOF mass spectra is a robust and convenient method of discriminating M. massiliense isolates from the other members of the M. abscessus complex.

Keywords: MALDI-TOF MS, VITEK MS, Mycobacteria, Identification, Nontuberculous mycobacterium

\section{Background}

Mycobacterium abscessus is a rapidly growing mycobacterium that is involved in soft-tissue and bone infections [1]. It is the most frequently isolated nontuberculous mycobacterium (NTM) found in European cystic fibrosis (CF) patients and has emerged as an important pathogen in CF lung disease [2, 3]. M. abscessus complex is split into three subspecies, $M$. abscessus (sensu stricto), $M$. massiliense, and M. bolletii, which are commonly separated by multilocus analysis of hsp65, rpoB, secA, and

\footnotetext{
* Correspondence: jan.kehrmann@uk-essen.de

${ }^{1}$ Institute of Medical Microbiology, University Hospital Essen, University

Duisburg-Essen, Essen, Germany

Full list of author information is available at the end of the article
}

the 16-23S internal transcribed spacer (ITS) region $[4,5]$. However, the nomenclature is still being debated; a recent proposal suggests uniting $M$. massiliense and M. bolletii to form M. abscessus subsp. bolettii comb. nov. and to distinguish that new combination from M. abscessus subsp. abscessus [6].

M. abscessus lung disease is difficult to treat, and the subspecies differ in antibiotic resistance [7]. The presence of an inducible methylase gene [erm (41)] in $M$. abscessus (sensu stricto) results in macrolide resistance under clarithromycin regimen therapy, while a functional $\operatorname{erm}(41)$ gene is missing in $M$. massiliense. This inducible resistance to clarithromycin is considered to explain the low efficiency of macrolide-containing 
therapy regimens in $M$. abscessus (sensu stricto) disease in contrast to the high treatment response rates in $M$. massiliense disease [8-10]. Furthermore, a rare mutation within the $r r l$ gene was reported to be responsible for macrolide resistance in $M$. abscessus complex [11]. Macrolides are the major therapeutic agents for M. massiliense, but the importance for $M$. abscessus (sensu stricto) is less clear. Many experts recommend a combination of parenteral agents based on in vitro MICs for macrolide resistant $M$. abscessus complex strains with amikacin and cefoxitin [10]. Due to the varying antibiotic therapy response of the different members of the M. abscessus complex, subspecies identification is of particular clinical interest. As their identification by using molecular methods and sequencing of several genes is cumbersome and not available in many laboratories, a routine diagnostic tool is desirable.

Several studies have used matrix-assisted laser desorption/ionization time-of-flight mass spectrometry (MALDI-TOF MS) to identify mycobacteria. However, this method currently cannot reliably differentiate the subspecies of $M$. abscessus complex [12-14]. The current versions of the Vitek MS databases (bioMérieux, Durham, NC, USA), Saramis v.4.12 (Vitek MS Plus, research use only [RUO]) and in vitro diagnostic (IVD 2.0), identify isolates at the level of the $M$. abscessus complex but do not separate M. abscessus (sensu stricto), M. massiliense, and M. bolletii. In the present study we determined the performance of Vitek MS Plus in discriminating the various subspecies by using the peak lists of the MALDI TOF mass spectra for principal component analysis (PCA), hierarchical cluster analysis (HCA), and single-peak analysis.

\section{Methods}

\section{Strains and cultures}

The study was performed in accordance with the Declaration of Helsinki and was approved by the ethics committee of the Medical School Hannover (protocol number 3023-2016). 50 pseudo-anonymized M. abscessus complex clinical isolates from the Department of Medical Microbiology and Hospital Epidemiology of the Hannover Medical School were used in this study; these isolates had been isolated from patient samples as part of standard care, collected as part of epidemiological surveillance and were characterized previously [15]. No written informed consent was necessary for this type of study. The identification of M. abscessus (sensu stricto) $(n=28), M$. massiliense $(n=19)$, and M. bolletii $(n=3)$ had been performed with sequencing of 16S rRNA and genotypic analysis of the $h s p 65$, erm(41), rrl, and rrs genes [15]. Isolates were grown from frozen stocks in mycobacterium growth indicator tube (MGIT, Becton, Dickinson and Company) liquid medium at $37{ }^{\circ} \mathrm{C}$ and were analyzed after 1 week of culture.

\section{Mycobacterial preparation protocol}

The mycobacterial preparation protocol suggested by bioMérieux was used with few modifications. We pipetted a $1.8-\mathrm{ml}$ aliquot of liquid medium from the bottom of the positive MGIT culture into a centrifuge vial and subjected it to centrifugation at $6,000 \times \mathrm{g}$ for $10 \mathrm{~min}$. The supernatant was discarded, and the pellet was resuspended in $500 \mu \mathrm{l}$ of $70 \%$ ethanol. After centrifugation at $6,000 \times \mathrm{g}$ for $10 \mathrm{~min}$, the pellet was resuspended in another $500 \mu \mathrm{l}$ of $70 \%$ ethanol and was transferred into a centrifuge vial containing $200 \mu \mathrm{l}$ of glass beads (Carl Roth GmbH \& Co., Karlsruhe, Germany), each $1 \mathrm{~mm}$ in diameter. The second ethanol washing step was added to the original preparation protocol of the manufacturer to improve removal of remnants. Furthermore, we used glass beads, $1 \mathrm{~mm}$ in diameter instead of $0.5 \mathrm{~mm}$ in diameter. The cells were disrupted with a vortex (MS3 digital shaker; IKA, Wilmington, NC, USA) for $15 \mathrm{~min}$ and then incubated at room temperature for $10 \mathrm{~min}$. The suspension was transferred into a sterile tube and subjected to centrifugation at $18,000 \times \mathrm{g}$ for $2 \mathrm{~min}$. The pellet was resuspended in $10 \mu \mathrm{l}$ of $70 \%$ formic acid. After the addition of $10 \mu \mathrm{l}$ of $100 \%$ acetonitrile, the suspension was briefly vortexed and subjected to centrifugation at $18,000 \times \mathrm{g}$ for $2 \mathrm{~min}$. Next, $1 \mu \mathrm{l}$ of the supernatant was pipetted onto the MALDI-TOF target slide. The samples were dried at room temperature, and $1 \mu \mathrm{l}$ of $\alpha$-cyano-4-hydroxy cinnamic acid (CHCA) matrix solution (bioMérieux, Marcy l'Etoile, France) was added. Samples were analyzed in quadruplicate after the matrix had crystallized at room temperature.

\section{Data processing}

Lists of the relative peak intensities (range, 2,000 $\mathrm{Da}$ to 20,000 Da mass-to-charge ratio $[\mathrm{m} / \mathrm{z}]$ ) derived from the raw data were used as input for HCA and PCA. We used the presets recommended by bioMérieux for smoothing, baseline subtraction, and peak detection. To compensate for small differences in the $\mathrm{m} / \mathrm{z}$ values of corresponding peaks across measurements that resulted from the limited accuracy of the measurement process itself and the calibration and peak-detection algorithm, we divided the recorded $\mathrm{m} / \mathrm{z}$ range from 2,000 $\mathrm{Da}$ to 20,000 Da into 3,600 bins of 5-Da widths, which is close to the mass accuracy assumed by the database-matching algorithm $(0.08 \%)$.

HCA and PCA, two separate cluster analytical methods, were performed with DataLab software (Epina $\mathrm{GmbH}$, Pressbaum, Austria). For HCA, Ward's method was used to calculate the Euclidean distance after standardization. HCA generates clusters by simply pairing individuals or subclusters that are the smallest distance apart in the space, with the original parameters as the coordinates (in our case, $\mathrm{m} / \mathrm{z}$ values). PCA is a 
type of multivariate analysis that reduces factors when there is redundancy in the data. It generates a new coordinate system by linear combination of the original coordinates ( $\mathrm{m} / \mathrm{z}$-values), which represent the principal components (PCs). This coordinate transformation is performed in such a way that the origin moves into the center of mass and the new coordinate axes are rotated so that they are now collinear to the principal axes of the data cloud. The PCs, as the new coordinates, are numbered according to the percentage of variance in the original data that they explain. The first three of the orthogonal and therefore linearly independent new coordinates (PCs 1, 2, and 3) should preferably be used to visualize the data as dots in a 3-dimensional space.

We cannot expect that the redundancy of the original 3,600 parameters is so great that the first three PCs alone will explain most of the total variance. Even so, it is possible to reveal substructures appearing as clusters, even if the number of involved original parameters $(\mathrm{m} /$ $\mathrm{z}$-values) that make up the difference is small, or if the differences themselves are small. PCA weights those parameters with a high loading factor, thereby amplifying their contribution to the new PCs to the disadvantage of parameters with only marginal variance. It is, simply speaking, this "amplification" that, in some settings, allows PCA to reveal reasonable clusters that are determined by small differences caused by the real grouping of individuals with predominantly similar parameters, something that HCA does not do. In such cases, using HCA is difficult because the differences in the Euclidean distances between individuals in the same group are comparable to those between individuals in other groups.

\section{Results}

All isolates included in the study were correctly identified as M. abscessus complex by analysis with Vitek MS Plus RUO software, which is based on SuperSpectra. Of the 50 isolates, 47 were identified with the highest possible confidence level (c.l.) of $99.9 \%$ (26 of 28 M. abscessus (sensu stricto) isolates, 18 of $19 \mathrm{M}$. massiliense isolates, and 3 of $3 \mathrm{M}$. bolletii isolates). The 3 remaining isolates were correctly identified with a c.l. of $94.5 \%$. Because the current versions of the VITEK MS databases (Saramis 4.12 (RUO) and IVD) cannot differentiate between the subspecies of the $M$. abscesssus complex (M. abscessus (sensu stricto), M. massiliense, and M. bolletii), we accessed the raw spectra and peak lists of all isolates by using the VITEK MS Plus RUO system to study unique differences between the subspecies by HCA, PCA, and single-peak analysis.

HCA using Ward's method, a commonly used approach, created several large mixed clusters with subclusters containing 2 to 6 isolates of a subspecies each; however, it did not reproduce the grouping according to the subspecies (data not shown).

PCA using the entire peak lists clearly separated all $M$. abscessus (sensu stricto) and M. massiliense isolates into two distinct clusters, visible in the 3-dimensional subspace spun by the first three principal components (Fig. 1). However, two isolates of M. abscessus (sensu stricto) were located in the border region of the M. massiliense cluster. The M. bolletii isolates did not form a separate cluster but were localized in the M. abscessus (sensu stricto) cluster (Fig. 1).

Furthermore, we performed single peak analysis of all isolates to detect unique differences within the three subspecies. The spectra of $M$. abscessus (sensu stricto) isolates and $M$. massiliense isolates differed substantially in two areas. The averaged spectra demonstrate specific M. massiliense peaks at 4,384 and $8,768 \mathrm{~m} / \mathrm{z}$, whereas these peaks were missing from the $M$. abscessus (sensu stricto) and the M. bolletii averaged spectra (Fig. 2). Otherwise, the averaged spectra of $M$. abscessus (sensu stricto) and $M$ bolletii isolates exhibited specific peaks at 4,390 and $8,781 \mathrm{~m} / \mathrm{z}$ that were absent from the averaged spectrum of $M$. massiliense (Fig. 2).

With a relative peak intensity of $1 \%$ as the threshold, single spectrum analysis demonstrated that the $4,384 \mathrm{~m} / \mathrm{z}$ peak was prevalent in $89.5 \%$ (17/19) of the M. massiliense isolates and the $8768 \mathrm{~m} / \mathrm{z}$ peak was present in $57.9 \%$ (11/19), whereas both peaks were absent from all $M$.

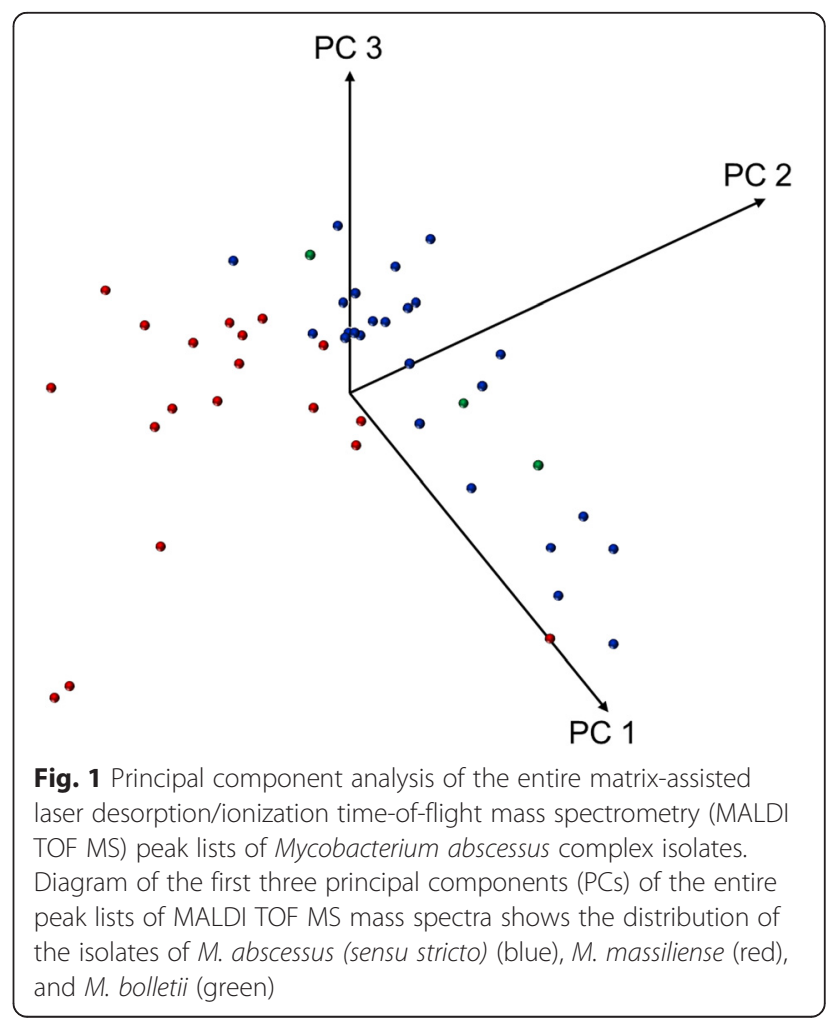



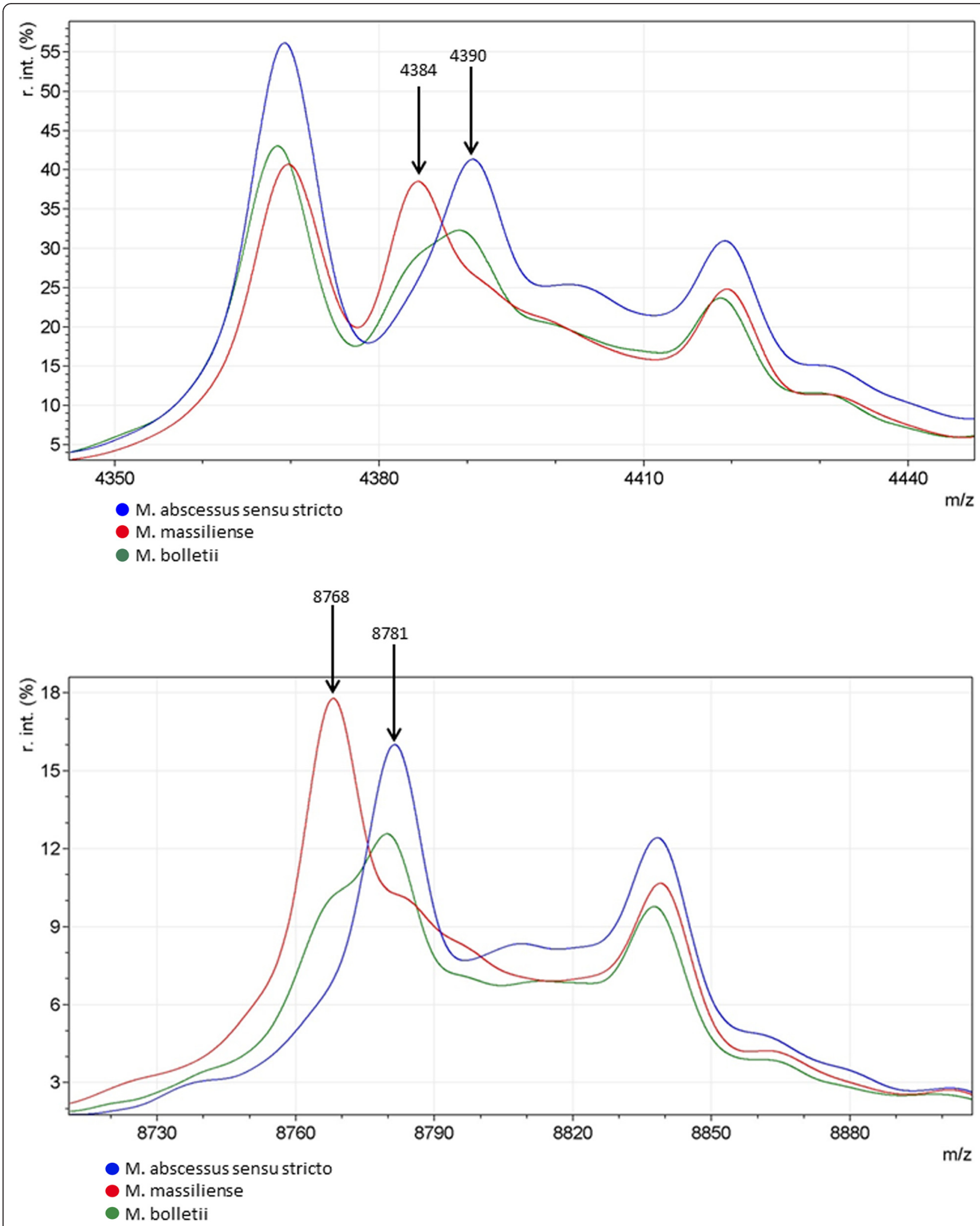

Fig. 2 Matrix-assisted laser desorption/ionization time-of-flight (MALDI TOF) averaged spectral profiles of discriminating peak regions of Mycobacterium abscessus complex isolates. Diagram shows relative intensity (\%) against mass-to-charge ratio ( $\mathrm{m} / \mathrm{z})$ values of the discriminating peak regions of MALDI TOF averaged mass spectral profiles for Mycobacterium abscessus (sensu stricto) (blue), M. massiliense (red), and M. bolletii (green) isolates 
abscessus (sensu stricto) and $M$. bolletii isolates (Table 1). The peak at $4,390 \mathrm{~m} / \mathrm{z}$ was present in all $M$. abscessus (sensu stricto) isolates $(28 / 28)$ and $M$. bolletii isolates (3/3), and the $8,781 \mathrm{~m} / \mathrm{z}$ peak was present in $89.2 \%(25 / 28)$ of $M$. abscessus (sensu stricto) isolates and $100 \%$ of the $M$. bolletii isolates, whereas the $4,390 \mathrm{~m} / \mathrm{z}$ peak was detected only in $5.2 \%(1 / 19)$ of $M$. massiliense isolates and the $8,781 \mathrm{~m} / \mathrm{z}$ peak was absent from all $19 M$. massiliense isolates (Table 1). No specific peak for M. bolletii was detected by the peak-detection algorithm with a $1 \%$ relative intensity threshold.

To analyze the relevance of the four discriminating peaks for separating $M$. abscessus (sensu stricto) isolates from $M$. massiliense isolates, we performed PCA by excluding these four peaks; nevertheless, we obtained a discrimination that was equally as good (Fig. 3) as that obtained with PCA including all peaks (Fig. 1). As Saramis 4.12 identifies $M$. abscessus at only the complex level, we examined whether a PCA restricted to the peaks included in the peak list of the $M$. abscessus SuperSpectra of the Saramis 4.12 database, supplemented with the discriminating peaks at 4,368 and $8,768 \mathrm{~m} / \mathrm{z}$, could distinguish $M$. massiliense and $M$. abscessus (sensu stricto). This procedure indeed separated both subspecies in two clusters, whereas $M$. bolletii isolates were also represented within the M. abscessus (sensu stricto) cluster (Fig. 4).

\section{Discussion}

In this study we examined the ability of VITEK MS Plus to differentiate the subspecies of the M. abscessus complex, comprising M. abscessus (sensu stricto), M. massiliense, and M. bolletii, cultured in MGIT liquid medium. We used MALDI TOF MS peak lists for HCA and PCA; we also analyzed the peak lists relating to differences between unique subspecies. Although HCA did not reproduce a grouping according to the subspecies, PCA clearly distinguished $M$. abscessus (sensu stricto) isolates and M. massiliense isolates. The difference in the results obtained with the two analytical methods can be explained by the different underlying algorithms used for data analysis. HCA performs clustering by calculating Euclidean distances between the isolates, resulting in

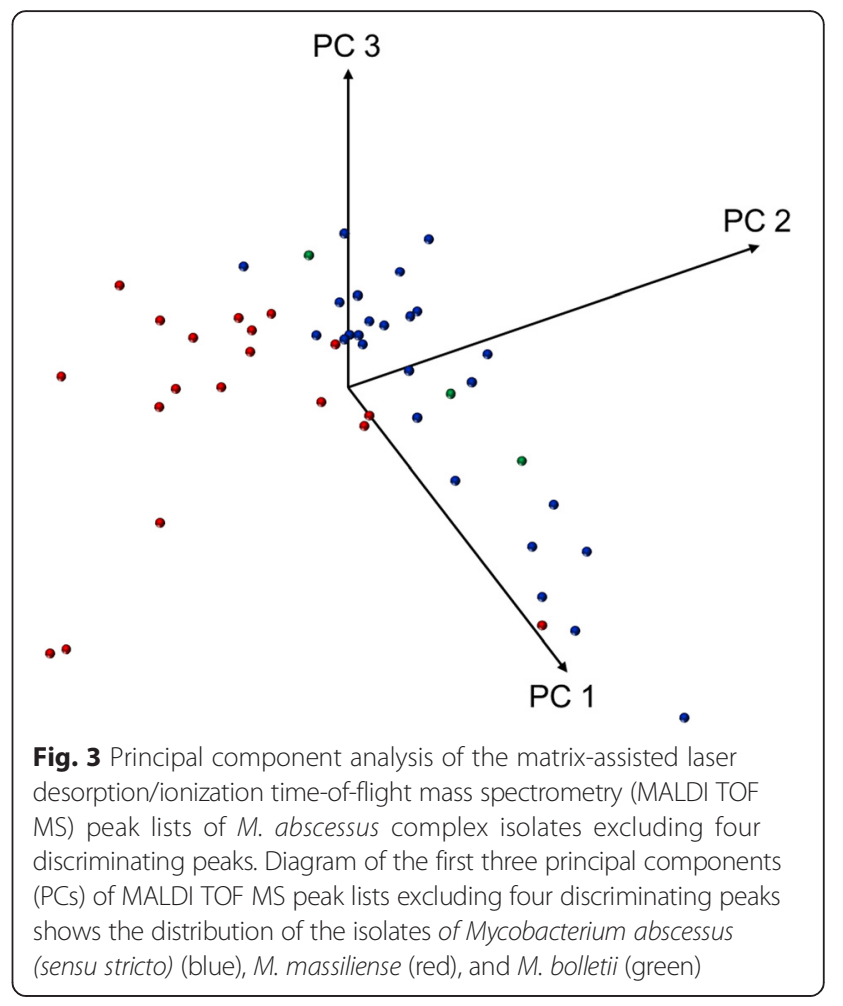

groups that exhibit multivariate similarity. In contrast, PCA finds those parameters that contribute to the maximal variance of the data and provide the best overall sample separation. In this way PCA reduces the number of dimensions for analysis and forms clusters that are based on inequalities [16, 17].

A previous study reported insufficient subspecies classification when entire peak lists were used for cluster analysis [18]. To the best of our knowledge, ours is the first study to use PCA as analytical method for discriminating the subspecies of the M. abscessus complex by MALDI TOF MS and to demonstrate that this analytical method is more useful than HCA for discriminating $M$. abscessus (sensu stricto) from $M$. massiliense. PCA from MALDI TOF MS peaks has been demonstrated to be an effective method of discriminating closely related bacterial or fungal species [19-22].

Comparison of average spectra demonstrated four peaks that were differentially present in discriminating

Table 1 Presence of the differentiating peaks in single spectra from matrix-assisted laser desorption/ionization time-of-flight mass spectrometry (MALDI TOF MS) among subspecies of Mycobacterium abscessus complex

\begin{tabular}{|c|c|c|c|c|}
\hline Peak m/z & 4384 & 4390 & 8768 & 8781 \\
\hline Subspecies & No $(\%)$ & No (\%) & No $(\%)$ & No $(\%)$ \\
\hline Mycobacterium abscessus (sensu stricto), $n=28$ & $0(0)$ & $28(100)$ & $0(0)$ & $25(89.3)$ \\
\hline Mycobacterium massiliense, $n=19$ & $17(89.5)$ & $1(5.3)$ & $11(57.9)$ & $0(0)$ \\
\hline Mycobacterium bolletii, $n=3$ & $0(0)$ & $3(100)$ & $0(0)$ & $3(100)$ \\
\hline
\end{tabular}




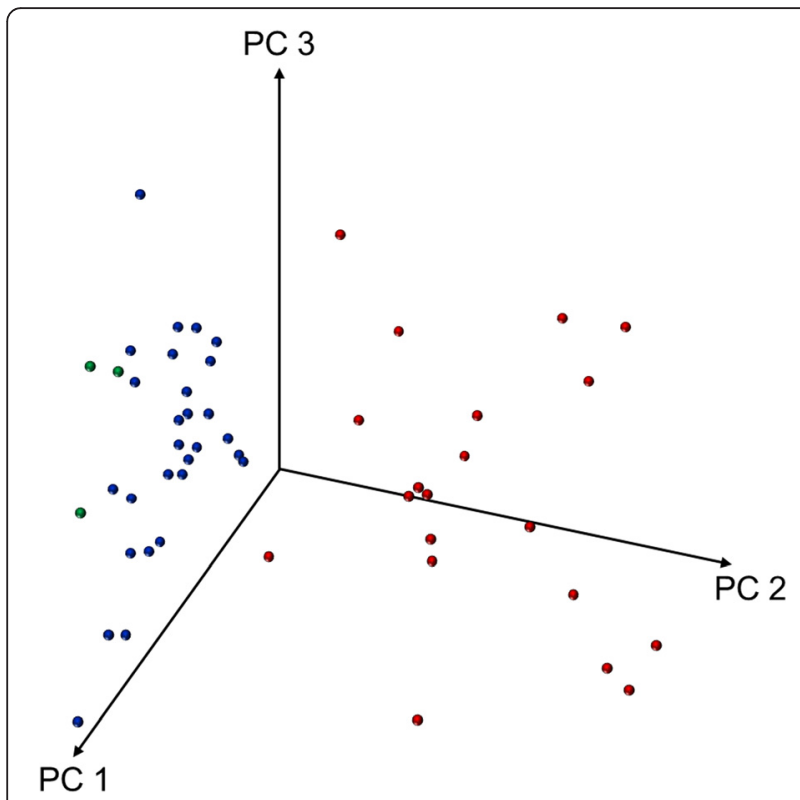

Fig. 4 Principal component analysis of the Mycobacterium abscessus complex SuperSpectrum supplemented with discriminating peaks. Diagram of the first three principal components (PCs) of preset $M$. abscessus SuperSpectrum of SARAMIS 4.12 software, supplemented with discriminating peaks identified in our study, shows the distribution of the isolates of $M$. abscessus (sensu stricto) (blue), M. massiliense (red), and M. bolletii (green)

M. abscessus (sensu stricto) from $M$. massiliense. It seems likely that these peaks form two pairs $(4384 / 8768$ and 4390/8781), in each case representing one molecule that is displayed twice in the peak spectrum, single charged on the one hand and double charged on the other. To date, three published studies have used singlepeak analysis to discriminate MALDI TOF MS peaks for members of the $M$. abscessus complex [18, 23, 24]. In two of these studies only two subspecies were discriminated [18, 23], and only one study analyzed isolates of all three subspecies [24], including $M$. bolletii, which is the most rarely isolated subspecies. However, none of those peaks accurately discriminated all of the isolates included in that study [24]. The authors speculated that the degree of horizontal gene transfer that occurs in the evolution of $M$. abscessus may be responsible for the limitations in accuracy [24].

Furthermore, most of the discriminating peaks described so far differed between these three studies and could not be confirmed by the other studies. The only peak determined to be discriminating in two of the three previous studies $[18,23]$ was detected at approximately the $8,769 \mathrm{~m} / \mathrm{z}$ value, which has been described as specific for M. massiliense. Our study also detected this specific peak, which showed $100 \%$ specificity for discriminating the isolates included in our study. The other peaks were described as discriminative by only one of the three studies and were not found in the other two. Two of the three peaks that we found in our study have been described by Teng et al. as discriminative for $M$. massiliense and M. abscessus (sensu stricto) [23]. The description of only a few discriminating peaks by these published studies indicates that the MALDI TOF MS proteome is strongly related across the three subspecies. This finding is in line with the strongly related genotype of the three subspecies, which makes multilocus sequencing necessary for clear discrimination.

A specific reason for the inability of these studies to reproduce the discriminating MALDI TOF MS peaks is unclear so far. The extraction protocols do not appear to play an important role, because two studies, that by Teng et al. [23] and that by Fangous et al. [24], applied exactly the same protocols and used the same MALDI TOF MS device. It has been speculated that differences in the biogeographic MS profiles of M. abscessus complex subspecies may play a role [24]. Furthermore, peaks with a low relative intensity, which have also been described to be discriminative and appear below a relative intensity of $1 \%$ [23, 24], may vanish after smoothing and baseline subtraction of the software, and therefore may not be confirmed by other studies. To avoid this issue, we considered only peaks with a relative intensity of at least $1 \%$ as candidates for a discriminative peak.

Restricting the discrimination between the $M$. abscessus subspecies to only one or very few peaks that do not seem to be reproducible by other studies does not appear to be an appropriate method. The use of PCA is more appropriate in this case, because PCA that excludes the four discriminating peaks detected in our study delineates the subspecies equally as well as PCA that includes these peaks. This finding indicates that the importance of these single peaks for subspecies identification is low when this analytical method is used.

Our study is limited by the small number of isolates used: 28 isolates of M. abscessus (sensu stricto), 19 isolates of $M$. massiliense, and 3 isolates of $M$. bolletii, which is rarely isolated. These small numbers may particularly influence discrimination with single-peak analysis, because bioregional variations may affect single MALDI TOF MS peaks more than they affect the entire peak spectrum.

\section{Conclusion}

In this study, we showed that PCA of MALDI TOF MS peaks is a robust method that separates $M$. massiliense from the other subspecies of the M. abscessus complex. This finding is particularly relevant for clinicians, because $M$. massiliense exhibits a better response to antibiotic treatment [9]. Supplementing the VITEK MS Plus 
identification system with a PCA-based, trained support vector machine or using linear discriminant analysis of the existing peak lists of $M$. abscessus complex in the Saramis 4.12 database, including the discriminating peaks found by our study, could help to distinguish $M$. massiliense isolates in routine microbiological diagnostics.

\section{Abbreviations}

c.l: confidence level; CF: cystic fibrosis; CHCA: a-cyano-4-hydroxy cinnamic acid; HCA: hierarchical cluster analysis; ITS: internal transcribed spacer; IVD: in vitro diagnostic; m/z: mass-to-charge ratio; MALDI-TOF MS: matrix-assisted laser desorption/ionization time-of-flight mass spectrometry; MGIT: mycobacterium growth indicator tube; NTM: nontuberculous mycobacterium; PCA: principal component analysis; RUO: research use only.

\section{Competing interests}

None of the authors has any potential financial or nonfinancial conflicts of interest related to this manuscript.

\section{Authors' contributions}

$\mathrm{JK}, \mathrm{FM}$, and JB conceived and designed the study; FB and AH provided the M. abscessus isolates; SW, RM, and JK carried out the laboratory work; FM, SW, and JK analyzed the data; and JK and FM wrote the paper. All authors read and approved the final manuscript.

\section{Acknowledgments}

No financial support was provided for this study.

\section{Author details}

${ }^{1}$ Institute of Medical Microbiology, University Hospital Essen, University Duisburg-Essen, Essen, Germany. ${ }^{2}$ Department of Medical Microbiology and Hospital Epidemiology, Hannover Medical School, Hanover, Germany.

Received: 3 September 2015 Accepted: 3 February 2016

Published online: 01 March 2016

\section{References}

1. Griffith DE, Aksamit T, Brown-Elliott BA, Catanzaro A, Daley C, Gordin F, et al. An official ATS/IDSA statement: diagnosis, treatment, and prevention of nontuberculous mycobacterial diseases. Am J Respir Crit Care Med. 2007; 175(4):367-416. doi:10.1164/rccm.200604-571ST.

2. Roux AL, Catherinot E, Ripoll F, Soismier N, Macheras E, Ravilly S, et al. Multicenter study of prevalence of nontuberculous mycobacteria in patients with cystic fibrosis in france. J Clin Microbiol. 2009:47(12):4124-8. doi:10.1128/JCM.01257-09.

3. Olivier KN, Weber DJ, Wallace Jr RJ, Faiz AR, Lee JH, Zhang Y, et al. Nontuberculous mycobacteria. I: multicenter prevalence study in cystic fibrosis. Am J Respir Crit Care Med. 2003;167(6):828-34. doi:10.1164/rccm. 200207-6780C.

4. Adekambi T, Berger P, Raoult D, Drancourt M. rpoB gene sequence-based characterization of emerging non-tuberculous mycobacteria with descriptions of Mycobacterium bolletii sp. nov., Mycobacterium phocaicum sp. nov. and Mycobacterium aubagnense sp. nov. Int J Syst Evol Microbiol. 2006;56(Pt 1):133-43. doi:10.1099/ijs.0.63969-0.

5. Adekambi T, Reynaud-Gaubert M, Greub G, Gevaudan MJ, La Scola B, Raoult $D$, et al. Amoebal coculture of "Mycobacterium massiliense" sp. nov. from the sputum of a patient with hemoptoic pneumonia. J Clin Microbiol. 2004; 42(12):5493-501. doi:10.1128/JCM.42.12.5493-5501.2004.

6. Leao SC, Tortoli E, Euzeby JP, Garcia MJ. Proposal that Mycobacterium massiliense and Mycobacterium bolletii be united and reclassified as Mycobacterium abscessus subsp. bolletii comb. nov., designation of Mycobacterium abscessus subsp. abscessus subsp. nov. and emended description of Mycobacterium abscessus. Int J Syst Evol Microbiol. 2011; 61(Pt 9):2311-3. doi:10.1099/ijs.0.023770-0

7. Kim HY, Kim BJ, Kook Y, Yun YJ, Shin JH, Kim BJ, et al. Mycobacterium massiliense is differentiated from Mycobacterium abscessus and Mycobacterium bolletii by erythromycin ribosome methyltransferase gene (erm) and clarithromycin susceptibility patterns. Microbiol Immunol. 2010;54(6):347-53. doi:10.1111/j.1348-0421.2010.00221.x.
8. Jeon K, Kwon OJ, Lee NY, Kim BJ, Kook YH, Lee SH, et al. Antibiotic treatment of Mycobacterium abscessus lung disease: a retrospective analysis of 65 patients. Am J Respir Crit Care Med. 2009;180(9):896-902. doi:10.1164/rccm.200905-07040C.

9. Koh WJ, Jeon K, Lee NY, Kim BJ, Kook YH, Lee SH, et al. Clinical significance of differentiation of Mycobacterium massiliense from Mycobacterium abscessus. Am J Respir Crit Care Med. 2011;183(3):405-10. doi:10.1164/rccm.201003-03950C.

10. Brown-Elliott BA, Nash KA, Wallace Jr RJ. Antimicrobial susceptibility testing, drug resistance mechanisms, and therapy of infections with nontuberculous mycobacteria. Clin Microbiol Rev. 2012;25(3):545-82. doi:10.1128/CMR.05030-11

11. Wallace Jr R, Meier A, Brown BA, Zhang Y, Sander P, Onyi GO, et al. Genetic basis for clarithromycin resistance among isolates of Mycobacterium chelonae and Mycobacterium abscessus. Antimicrob Agents Chemother. 1996:40(7):1676-81.

12. Saleeb PG, Drake SK, Murray PR, Zelazny AM. Identification of mycobacteria in solid-culture media by matrix-assisted laser desorption ionization-time of flight mass spectrometry. J Clin Microbiol. 2011;49(5):1790-4. doi:10.1128/ JCM.02135-10.

13. Mather CA, Rivera SF, Butler-Wu SM. Comparison of the Bruker Biotyper and Vitek MS matrix-assisted laser desorption ionization-time of flight mass spectrometry systems for identification of mycobacteria using simplified protein extraction protocols. J Clin Microbiol. 2014;52(1):130-8. doi:10.1128/JCM.01996-13.

14. Pignone M, Greth KM, Cooper J, Emerson D, Tang J. Identification of mycobacteria by matrix-assisted laser desorption ionization-time-of-flight mass spectrometry. J Clin Microbiol. 2006;44(6):1963-70. doi:10.1128/JCM.01959-05.

15. Ruger K, Hampel A, Billig S, Rucker N, Suerbaum S, Bange FC. Characterization of rough and smooth morphotypes of Mycobacterium abscessus isolates from clinical specimens. J Clin Microbiol. 2014;52(1):244-50. doi:10.1128/JCM.01249-13.

16. Jurs PC. Pattern recognition used to investigate multivariate data in analytical chemistry. Science. 1986;232(4755):1219-24.

17. Fiehn O, Kopka J, Dormann P, Altmann T, Trethewey RN, Willmitzer L. Metabolite profiling for plant functional genomics. Nat Biotechnol. 2000;18(11):1157-61. doi:10.1038/81137.

18. Panagea T, Pincus DH, Grogono D, Jones M, Bryant J, Parkhill J, et al. Mycobacterium abscessus Complex Identification with Matrix-Assisted Laser Desorption Ionization-Time of Flight Mass Spectrometry. J Clin Microbiol. 2015;53(7):2355-8. doi:10.1128/JCM.00494-15.

19. Zautner AE, Masanta WO, Tareen AM, Weig M, Lugert R, Gross U, et al. Discrimination of multilocus sequence typing-based Campylobacter jejuni subgroups by MALDI-TOF mass spectrometry. BMC Microbiol. 2013;13:247. doi:10.1186/1471-2180-13-247.

20. Niyompanich S, Jaresitthikunchai J, Srisanga K, Roytrakul S, Tungpradabkul S Source-identifying biomarker ions between environmental and clinical Burkholderia pseudomallei using whole-cell matrix-assisted laser desorption/ ionization time-of-flight mass spectrometry (MALDI-TOF MS). PLoS One. 2014;9(6):e99160. doi:10.1371/journal.pone.0099160.

21. Dolatabadi S, Kolecka A, Versteeg M, de Hoog SG, Boekhout T. Differentiation of Clinically Relevant mucorales Rhizopus microsporus and R. arrhizus by Matrix-Assisted Laser Desorption lonization-Time of Flight Mass Spectrometry (MALDI-TOF MS). J Med Microbiol. 2015. doi:10.1099/jmm.0.000091.

22. Vidigal PG, Mosel F, Koehling HL, Mueller KD, Buer J, Rath PM, et al. Delineation of Stenotrophomonas maltophilia isolates from cystic fibrosis patients by fatty acid methyl ester profiles and matrix-assisted laser desorption/ionization time-of-flight mass spectra using hierarchical cluster analysis and principal component analysis. J Med Microbiol. 2014:63(Pt 12):1615-20. doi:10.1099/jmm.0.076950-0.

23. Teng SH, Chen CM, Lee MR, Lee TF, Chien KY, Teng LJ, et al. Matrix-assisted laser desorption ionization-time of flight mass spectrometry can accurately differentiate between Mycobacterium masilliense (M. abscessus subspecies bolletti) and M. abscessus (sensu stricto). J Clin Microbiol. 2013;51(9):3113-6. doi:10.1128/JCM 01239-13.

24. Fangous MS, Mougari F, Gouriou S, Calvez E, Raskine L, Cambau E, et al. Classification algorithm for subspecies identification within the Mycobacterium abscessus species, based on matrix-assisted laser desorption ionization-time of flight mass spectrometry. J Clin Microbiol. 2014;52(9):3362-9. doi:10.1128/JCM.00788-14. 